\title{
NUMERICAL INVESTIGATION OF TANGENTIAL BLOWING OVER THE RUDDER OF A VERTICAL TAILPLANE
}

\author{
A. Gebhardt, German Aerospace Center (DLR), Aerodynamics and Flow \\ Technology, Lilienthalplatz 7, 38108 Braunschweig, Germany
}

\begin{abstract}
On a vertical tailplane tangential blowing over the shoulder of a deflected rudder is applied. With large rudder deflection angle and without blowing the flow is mostly separated on the rudder. Three geometries are investigated in a numerical study with the aim to increase the side force coefficient. The first one is the baseline configuration without blowing, second a geometry with a continuous full span slot and third a geometry where discrete slots are used. With the continuous slot the separation on the rudder can be avoided completely while it is greatly reduced for the discrete slots. This is investigated for different sideslip angles. An approximately linear increase in the side force coefficient can be found until a sudden side force breakdown occurs. In a further study, the jet blowing velocity is varied. A smaller jet velocity leads to a smaller increase in the side force coefficient. Comparing the continuous and discrete slot configurations shows, that for a similar increase in the side force coefficient a much smaller mass flow rate is needed for the discrete slots. However, for jet velocities below the sonic speed the increase in the side force coefficient is limited. It can only be increased by a larger slot width in span wise direction but this comes at the expense of an increase in the required mass flow rate.
\end{abstract}

\section{INTRODUCTION}

Active flow control is investigated for a vertical tailplane (VTP) geometry as one of the promising applications of this technology. The vertical tailplane of a transport aircraft is needed for stability and control of the aircraft about the yaw axis. One case determining the size of the VTP is the failure of the critical engine which is usually outside the symmetry plane. In this one-engine inoperative (OEI) condition, the resulting asymmetric thrust creates a moment around the yaw axis. This must be counteracted by the VTP. Here take-off is a critical flight segment. The flight speed is relatively low and thus also the aerodynamic forces produced by the VTP but the thrust of the engines is high and with this the resulting moment for the OEI condition. On the other hand, in cruise flight, which makes up most of the flight time, the aircraft velocity is high. In this flight condition the VTP is larger than necessary to satisfy stability requirements for modern transport aircraft with electronic flight contro systems. This means, that the VTP size is determined by a rarely occurring failure case. In this critical OEI case the rudder is highly deflected to achieve the required large side force but this also leads to partial flow separation on the rudder. If the size of the VTP could be reduced through the application of some means to increase the side force, drag and weight could be lowered which in turn would lead to a reduction in fuel burn. One possibility is the use of active flow control (AFC). With this the side force coefficient produced by the VTP can be increased without increasing the VTP size by delaying flow separation to higher sideslip and/or rudder deflection angles.

In contrast to passive flow control using for example vortex generators, active flow control has the possibility to be turned on just when needed. On the other hand some source of energy is needed for the AFC system which is not the case for a passive system. For this investigation, pressurized air is used as the energy source to drive the actuators. These type of actuators is already used in a wide range of active flow control applications. Among different kinds of blowing techniques tangential blowing over the rudder is selected with the jet exit being located at the end of the fin.

Tangential blowing has already been investigated experimentally and numerically in several studies but mainly for the use on wings $[1,2]$. In contrast to the wing, the VTP has a small aspect ratio and a large sweep angle changing its flow characteristics. The flow is more three dimensional (3D) for the VTP. Thus the results obtained for the wing might not be directly transferable. Concerning AFC application to the VTP, some recent studies were done at NASA and Boeing with a mainly experimental approach [3-7]. However, for these studies different kinds of actuators were used, namely synthetic jets and sweeping jets, with a different and specific effect on and interaction with the flow field. Some numerical investigations were done using the code Overflow. Substantial computational resources were applied to demonstrate the ability to simulate sweeping jets on a 3D vertical tail [8]. In the present study a different flow solver as well as a different kind of blowing technique will be used and applied to the 3D VTP geometry with deflected rudder.

Typically tangential blowing is seen as a brute force method. It requires a large amount of compressed air but can also lead to a large increase in the lift or side force coefficient. In a common approach, a blowing slot extends over the whole or at least large parts of the span. In the following discussion, this setup will be investigated as well and will serve as a reference. In addition, to reduce the required air mass flow rate, the effect of having just part of the span affected with blowing slots is investigated. Therefore the full span slot is divided into several discrete 
slots with finite width and some gaps without slots between them. The slot width and gap size was taken from a previous investigation of an infinite swept wing with a constant chord [9]

In the following, a comparison of the results with the continuous and the discrete slots will be presented. In addition, to enhance the understanding of the discrete slot configuration, the blowing jet velocity is varied to see the effect on the flow and the side force coefficient. Furthermore, a variation of the sideslip angle is investigated since it is usually not zero for the scenario of a one-sided engine failure.

\section{GEOMETRY SETUP}

The geometry consists of a VTP of a conventional transport aircraft which has a typical symmetric airfoil. The forward fixed part of the VTP is called fin and the deflectable aft part rudder. The hinge-line about which the rudder is rotated is located at $67 \%$ of the local chord length. The side view of the VTP is given in FIG. 1. In this figure the rudder is deflected away from the observer point of view. A rudder deflection angle of $30^{\circ}$ is used for the investigation results presented.

The leading edge sweep angle of the VTP is $44^{\circ}$ and the trailing edge sweep angle $25^{\circ}$. The geometry is scaled to wind tunnel size and features a span of $0.8502 \mathrm{~m}$. In a later stage of the investigation it is planned to compare the numerical results to wind tunnel results.
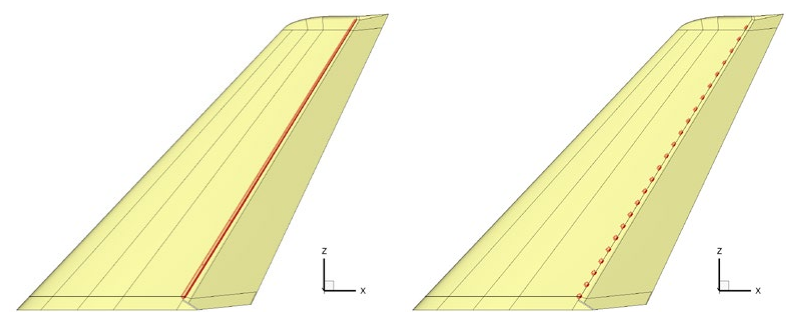

FIG. 1: Side view of the VTP with deflected rudder; the red color shows the two types of slot geometries investigated: left: full span slot, right: discrete slots

The blowing slot, from which air is blown tangentially over the deflected rudder, is located at the end of the fin. A slice through the geometry is shown in FIG. 2. The slot height is 0.0006 of the local chord length leading to a relatively thin channel. This height was also used for the preceding investigations with an infinite swept wing [9] For a middle VTP section this would be a slot height of $0.32 \mathrm{~mm}$. A part of the slot is modelled for numerical reasons [10]. The slot length corresponds to 20 times its height and is chosen so that a developed pipe flow is established at the outlet.

The first geometry features a continuous slot going from root to the tip of the VTP. This geometry is shown in FIG. 1 on the left side with the red line visualizing the location of the slot. On the right side the discrete slot configuration is depicted with 24 slots. Each slot has a width in spanwise direction of $10 \mathrm{~mm}$ and a gap to the next slot of $30 \mathrm{~mm}$.

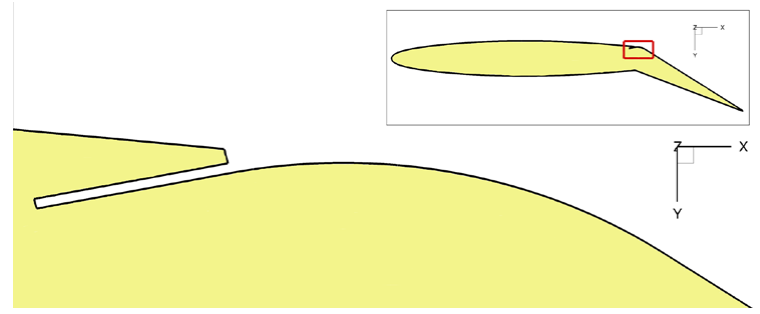

FIG. 2: Detail of the blowing slot geometry

\section{MESH GENERATION}

For the mesh generation the commercial software Centaur by CentaurSoft is used [11] which creates hybrid meshes. The surface mesh consists of triangles or quadrilaterals. The near surface mesh is filled with prisms or hexahedrons and the remainder of the flow field with tetrahedrons. The slots itself and also the rudder are meshed with quadrilaterals or hexahedrons where ever possible. In addition, the flow field behind and above the rudder is discretized with hexahedrons which are less dissipative and should lead to a better preservation of the flow quantities. In this area separated flow for the cases without or using insufficient blowing or vortices due to the blowing jets are expected. A slice through the vertical tailplane section and its surrounding mesh is given in FIG. 3.

The number of cell layers used to resolve the boundary layer is 50 with the target dimensional first wall distance $\mathrm{y}^{+}$ of 0.5 . The $\mathrm{y}^{+}$is selected smaller than usual for the used turbulence model to better capture the high velocity jet which is close to the rudder surface in the vicinity of the rudder shoulder. Especially around the VTP the flow field is refined. This leads in total to a mesh size of about 70 million nodes. Due to the very small height of the slot the number of layers is reduced inside the channel. Outside the layers are growing rapidly as depicted in FIG. 4.

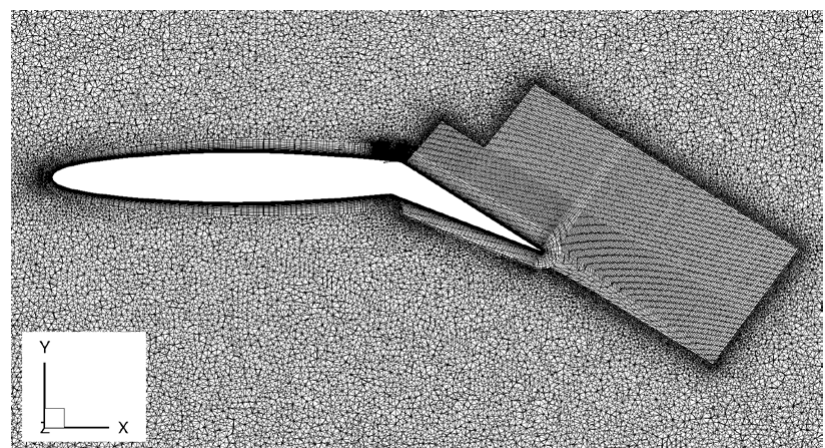

FIG. 3: Mesh with hexahedron field block refinement above and behind the rudder 


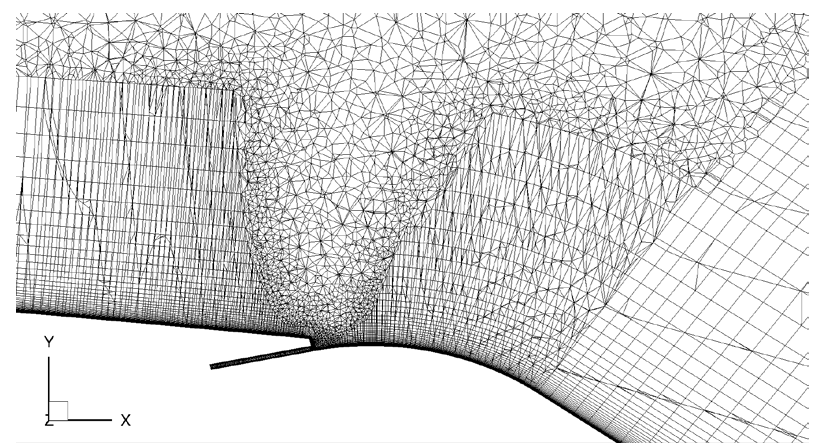

FIG. 4: Mesh in the vicinity of the slot

\section{FLOW SIMULATION}

The flow computations were carried out with the flow solver TAU, release 2016.1.0, developed by DLR (Deutsches Zentrum für Luft- und Raumfahrt, German Aerospace Center) [12].

With TAU the three dimensional Reynolds-averaged Navier-Stokes (RANS) equations are solved. A finite volume method is applied for the spatial discretization. The temporal discretization is achieved by a semi-implicit Backward-Euler scheme with the linear LUSGS (LowerUpper Symmetric Gauss-Seidel) solver. The inviscid flux discretization type for the first stage of iterations is a first order upwind scheme, thereafter switching to the central scheme introduced by Jameson. A matrix dissipation scheme is employed for low numerical dissipation. For convergence acceleration a $3 w$ multigrid scheme is used. The Reynolds number is $\operatorname{Re}=1.88 \times 10^{6}$ based on the VTP airfoil mean aerodynamic chord at an onflow velocity of $57 \mathrm{~m} / \mathrm{s}$.

The viscous, fully turbulent RANS calculations are performed using the turbulence model of Spalart and Allmaras [13] enhanced with a vortical and rotational flow correction (SARC) based on the approach of Spalart and Shur [14]. For circulation control airfoils it was shown that this turbulence model leads to good results for flows with high streamline curvature [15]

For the calculations with blowing activated, an actuation boundary condition is specified at the upstream wall of the slot to inject the jet flow into the flow domain. For this boundary condition a specification of jet velocity and density is necessary, with the latter assumed to be identical to the value of the flow in the farfield. Steady RANS calculations are performed with a constant blowing jet.

When comparing the results of the flow simulations, the mass flow rate will used as a measure of how much pressurized air has to be provided by the system from some external source. The mass flow rate is defined by

(1) $\dot{m}_{j}=v_{j}^{*} \rho_{j}^{*} A_{j}$

where $v_{j}$ is the jet velocity, $\rho_{j}$ is the jet density and $A_{j}$ is the area of the slot exit.

\section{RESULTS}

In this chapter the results will be presented starting with an overview of the baseline flow. Then the effect of tangential blowing and the impact of changing the continuous slot to discrete slots will be explained. Thereafter the resulting side force coefficients will be compared for different sideslip angles $\beta$. This corresponds to the lift coefficient development for changing angles of attack for a normal aircraft wing, but, since the vertical tailplane is rotated with respect to the wing orientation, the relevant angle variation is that of the sideslip angle. The characteristic flow effects will be discussed and the impact of a variation of the jet velocity will be analyzed.

\subsection{Baseline Flow}

The baseline flow without any active flow control device activated is studied for a case with a large rudder deflection angle of $30^{\circ}$. This leads to partly separated flow on the rudder. In FIG. 5 the areas with a negative skin friction coefficient are shown, i.e. regions, where a flow component opposite to the onflow direction exists. This is an indication for separated flow. On the fin the stream traces are approximately parallel to the onflow direction. Due to the large rudder deflection the flow cannot follow the rudder surface on its suction side. The flow component in onflow direction is decelerated so that the component in spanwise direction prevails. Due to this the stream traces on large parts of the rudder point in spanwise direction. This is already the case for no or small sideslip angles, which the results shown in the figure represent.

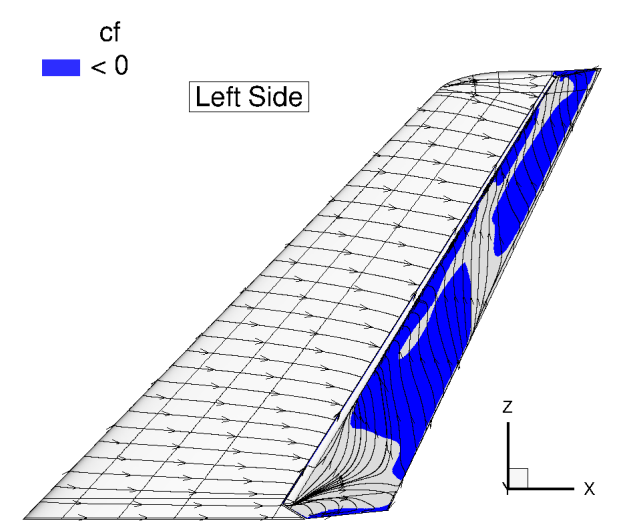

FIG. 5: Skin friction coefficient and stream traces for the configuration without blowing, pressure side, $\beta_{1}$

Looking at the pressure coefficient surface distribution in FIG. 6 it can be observed, that only in the lower part the rudder shoulder shows the characteristic suction peak with the red color. This confirms that the flow is weakened especially in the upper part of the rudder and is mostly separated there. Also the field stream traces in FIG. 7, which are tracked close to the surface, support this. As they cross the hinge line from the fin to the rudder, they move away from the surface and underneath them the spanwise flow from root to tip is made visible by the stream traces. 


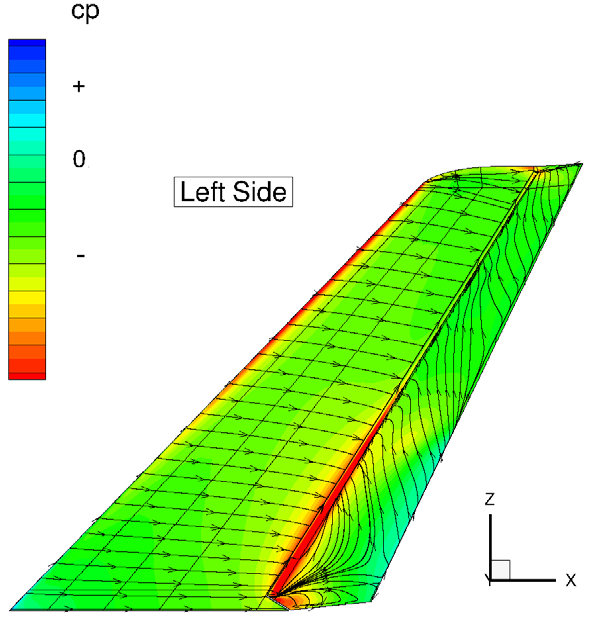

FIG. 6: Surface pressure coefficient and stream traces for the configuration without blowing, suction side, $\beta_{1}$

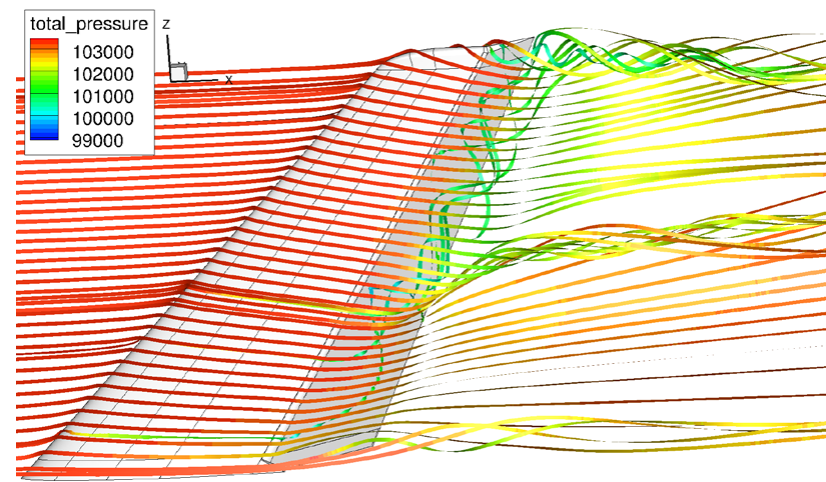

FIG. 7: Field stream traces colored with total pressure for the configuration without blowing, suction side, $\beta_{1}$

\subsection{Effects of Tangential Blowing}

Through the use of tangential blowing the aim is to reduce the areas of separated flow and to increase the side force coefficient. A small jet with a velocity higher than that of the incoming flow is injected close to the rudder surface at the end of the fin as shown in FIG. 1 on the left side. This jet adds energy to the flow and helps to overcome the adverse pressure gradient. Due to this the flow stays attached to the surface longer.

The effect on the VTP can be observed in FIG. 8 where a quite high jet velocity of $300 \mathrm{~m} / \mathrm{s}$ is applied on the full span slot. It can be seen that no blue areas of negative skin friction coefficient, i.e. of reversed flow, exist in contrast to FIG. 5. In addition, the stream traces on the rudder no longer indicate a root-to-tip spanwise flow. More specific, they are oriented about perpendicular to the hinge line. This is due to the fact that the blowing of the air is perpendicular to the hinge line too.

Comparing the baseline flow result in FIG. 6 with the flow in FIG. 9 where blowing is activated also shows a significant change in the surface pressure distribution. With blowing at the end of the fin activated, the pressure is reduced at the rudder shoulder over the entire span and leads to a pronounced suction peak. Although the blowing is just applied at the end of the fin, the leading edge of the fin also shows an increase in the pressure peak due to the upstream effect of the flow.

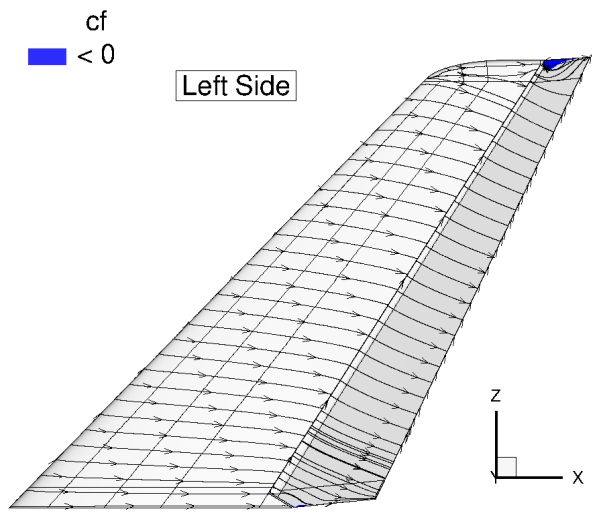

FIG. 8: Skin friction coefficient and stream traces, configuration with blowing, suction side, $v_{j}=300 \mathrm{~m} / \mathrm{s}$, continuous slot, $\beta_{1}$

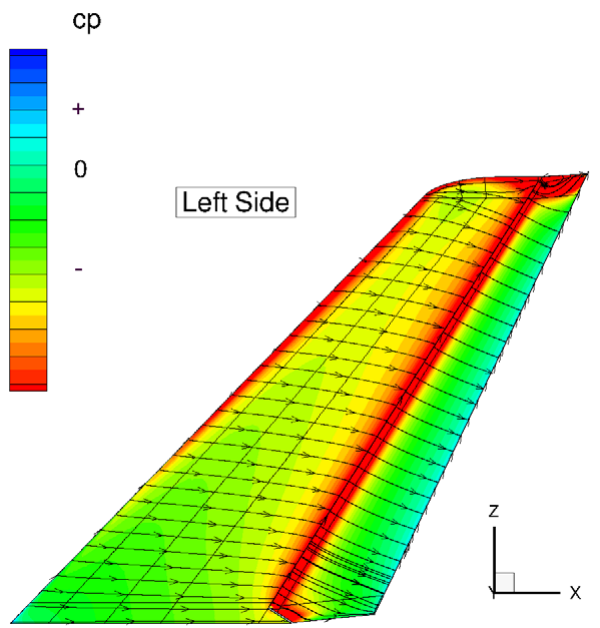

FIG. 9: Surface pressure coefficient and stream traces, configuration with blowing, suction side, $v_{j}=300 \mathrm{~m} / \mathrm{s}$, continuous slot, $\beta_{1}$

\subsection{Effects of Discrete Slots}

Exchanging the full span slot with several discrete slots and actuating with the same jet velocity leads to a surface flow topology as shown in terms of the skin friction coefficient in FIG. 10 and in terms of the surface pressure coefficient in FIG. 11. It is visible in FIG. 10 that now some small areas of reversed flow exist on the rudder. In the lower part of the rudder towards the root these regions are very small. They are close to the rudder shoulder and are located between the jets. In the upper part the areas of reversed flow increase somewhat and show a more irregular pattern. Particularly where the separated areas are the largest an increase in the pressure coefficient in FIG. 11 can be found, i.e. the rudder shoulder suction peak is reduced. Also the streamlines are a bit more oriented towards the tip compared to FIG. 9. 


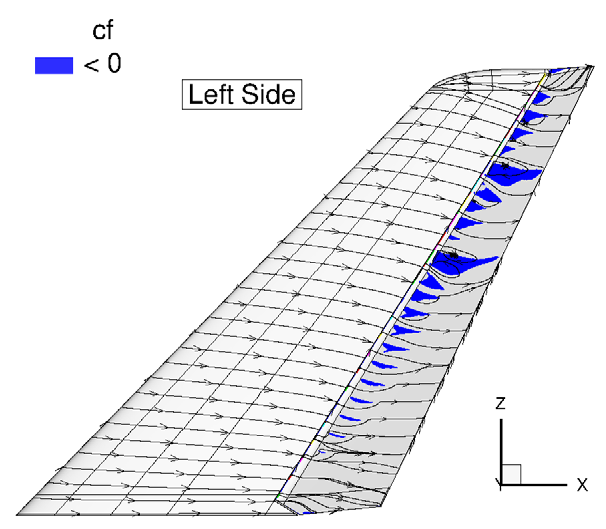

FIG. 10: Skin friction coefficient and stream traces, configuration with blowing, suction side, $v_{j}=300 \mathrm{~m} / \mathrm{s}$, discrete slots, $\beta_{1}$

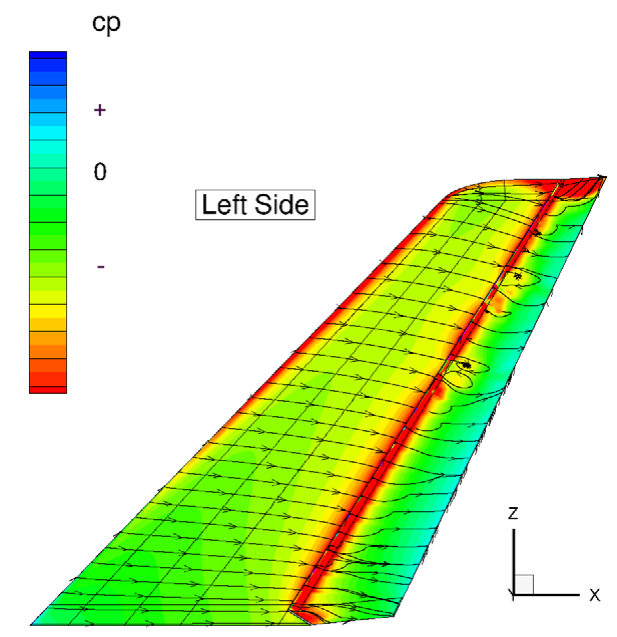

FIG. 11: Surface pressure coefficient and stream traces, configuration with blowing, suction side, $v_{j}=300 \mathrm{~m} / \mathrm{s}$, discrete slots, $\beta_{1}$

When looking in the vorticity distribution in the near-wall region, a major difference can be seen in FIG. 12 between the continuous and discrete slots impact on the flow. In FIG. 12a) the view is from behind the VTP onto the rudder. The continuous slot shows no vortices except the vortex at the root and at the tip which are also present for the configuration without blowing. In FIG. 12b) longitudinal vortices are present on the rudder. They are regular in the lower part of the VTP and increased and more irregular in the top part. This corresponds to the negative surface friction coefficient areas in FIG. 10 which are also more irregular in the upper part. The field slices on the rudder are colored for the visualization of the vortices with $\omega_{x}$, the rotation around the $x$-axis. From the observer point of view the blue vortices, with a negative $\omega_{x}$, rotate clockwise and the red ones counter-clockwise. A zoom of the root section of FIG. 12b) is given in FIG. 13. Here it can be observed that at each slot a counter rotating vortex pair is created. In addition the stream traces in the vicinity of one slot are depicted. From the slot the jet is going perpendicular to the hinge-line downstream the rudder. This blowing direction has an angle to the incoming flow from the fin. Where both flow directions meet, some shearing is induced. This leads to the creation of the blue vortex and the counter rotating red vortex below that. The creation of the vortices is possible, since the slot is not continuous and the flow from the fin has room to go between the jets due to the distance of adjacent slots. a) Continuous slot

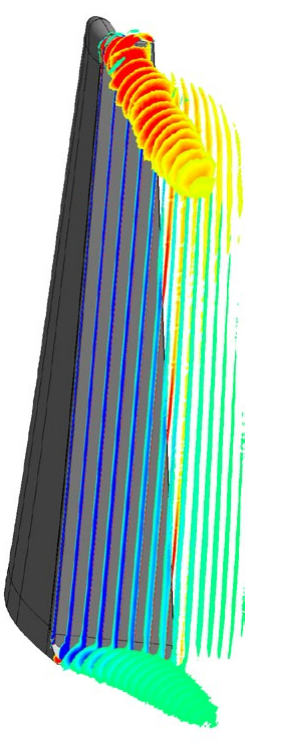

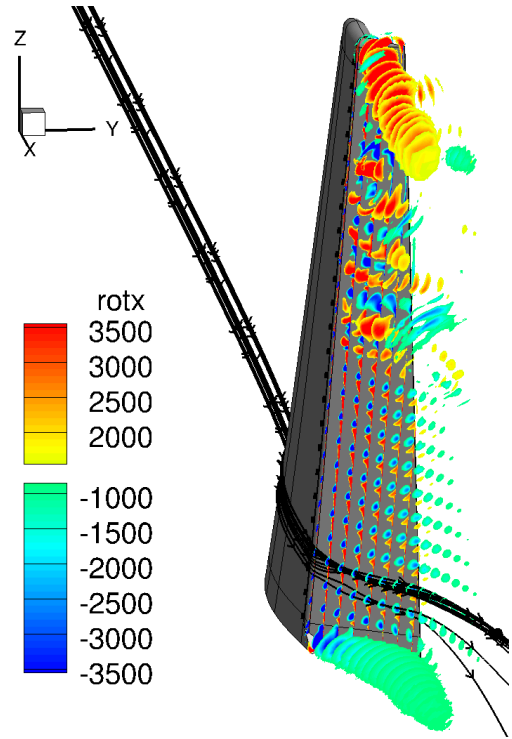

b) Discrete slots
FIG. 12: Field slices showing the vortices on the rudder, aft view, $v_{\mathrm{j}}=300 \mathrm{~m} / \mathrm{s}, \beta_{1}$

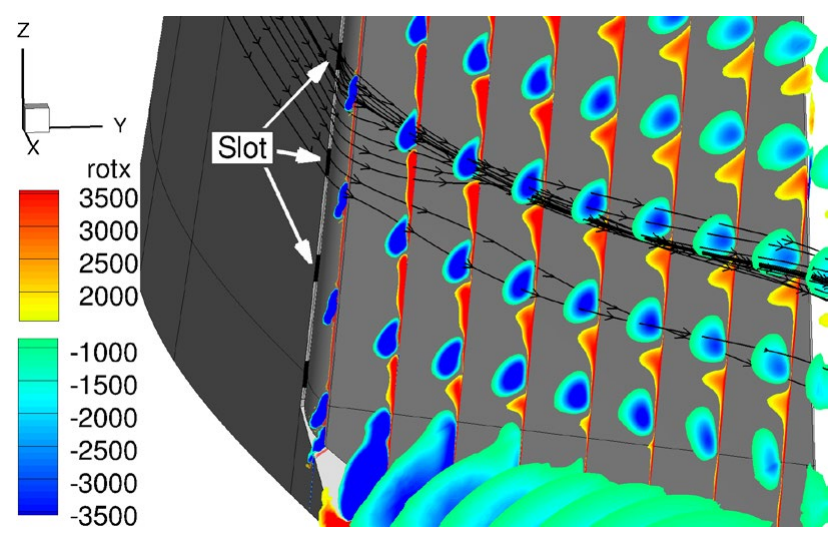

FIG. 13: Stream traces and field slices showing the vortices, aft view, zoom rudder root, $v_{j}=300 \mathrm{~m} / \mathrm{s}$, discrete slots, $\beta_{1}$

Where the upper blue and the lower red vortex meet, an upwind region is created where fluid is transported away from the surface. This is locally disadvantageous. However, on the other side where the vortices are rotating towards the surface, they introduce additional energy into the near surface flow, also affecting the regions not directly influenced by the jets. Thus, in addition to the energy added to the flow by the tangentially blown jets, the vortices also help to energize the flow and keep it attached in large parts of the rudder. In the upper part of the VTP the energy added by the jet is not enough to keep the flow attached everywhere. Due to this some 
increased areas of reversed flow between the jets can be found in FIG. 11.

\subsection{Variation of the Sideslip Angle}

The above results were presented for a small sideslip angle. Since for the critical one engine inoperative flight condition the sideslip angle can vary, higher angles are also investigated. The results will be presented in this section.

\subsubsection{Side Force Coefficient}

In FIG. 14 the side force coefficient $\Delta \mathrm{C}_{\mathrm{s}}$ is given versus a variation of the sideslip angle $\beta$. The side force coefficient is given as an increment and referenced to the calculation result for the configuration without blowing for the smallest sideslip angle $\beta_{1}$. The force coefficients are obtained by integration of the surface pressure and friction drag excluding the jet boundary plane.

The figure shows that with increasing sideslip angle the side force coefficient increases linearly. This is the same behavior known from wings. After $\beta_{3}$ an abrupt side force breakdown can be observed. Comparing the different curves they exhibit an approximately parallel shift to higher $\Delta \mathrm{C}_{\mathrm{s}}$. The discrete slots lead to a significant increase in the side force coefficient compared to the baseline case with no AFC. This performance improvement magnitude nearly doubles for the continuous slot results. The angle where the side force breakdown occurs stays the same for the baseline and the continuous slot configuration, but is shifted by $1^{\circ}$ for the discrete slot case.

The side force breakdown occurs due to a separation starting at the tip of the fin which increases at $\beta_{4}$, rapidly leading to a separation on the upper part of the fin. This is shown in FIG. 15 for the baseline configuration without blowing. Here the design of the VTP tip section might not be ideal for larger sideslip angle. This phenomenon is the same if blowing over the rudder is applied as shown for the continuous slot in FIG. 16 and for the discrete slots in FIG. 17. What changes in this case is a larger area of attached flow on the rudder. This is also the reason why the side force coefficient is somewhat higher at $\beta_{5}$ for the configurations with blowing activated. For the continuous slot the rudder is completely attached while for the discrete slots just directly in the jet path the flow can be attached leaving separated areas between them.

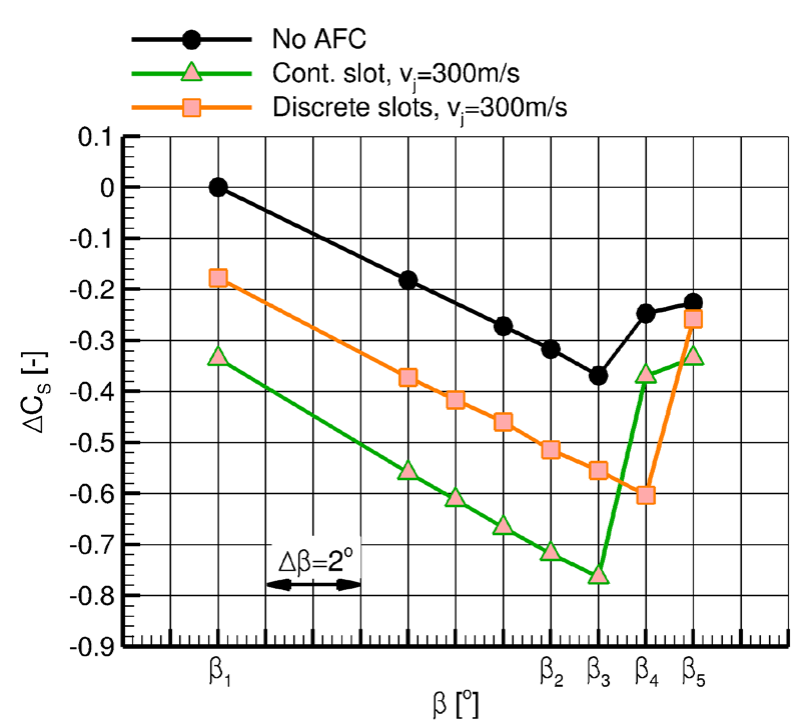

FIG. 14: Side force coefficient versus the sideslip angle, $v_{\mathrm{j}}=300 \mathrm{~m} / \mathrm{s}$

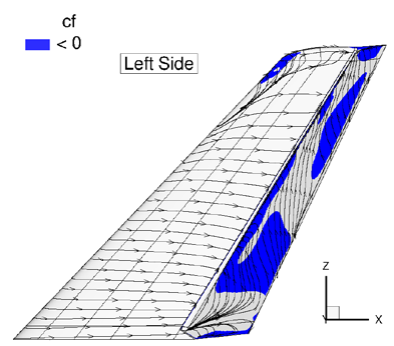

a) $\beta_{3}$

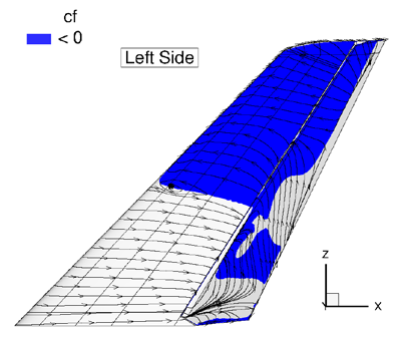

b) $\beta_{4}$
FIG. 15: Skin friction coefficient and stream traces, configuration without blowing, suction side

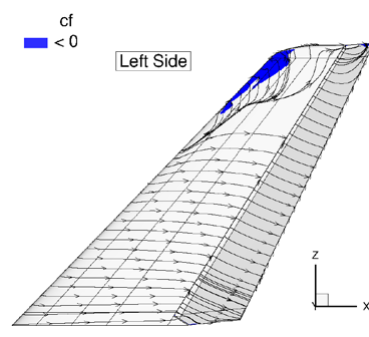

a) $\beta_{3}$

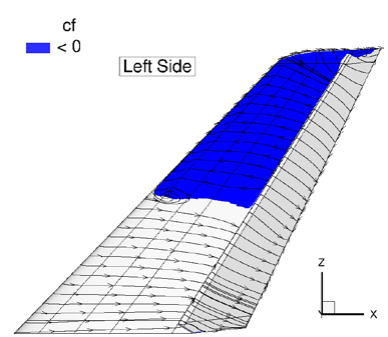

b) $\beta_{4}$
FIG. 16: Skin friction coefficient and stream traces, configuration with blowing, suction side, $\mathrm{v}_{\mathrm{j}}=300 \mathrm{~m} / \mathrm{s}$ continuous slot 


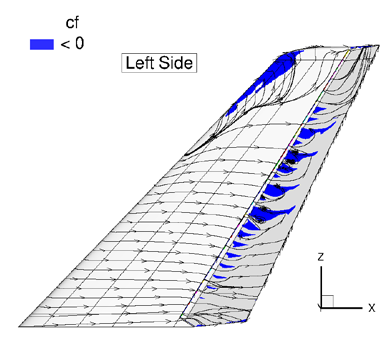

a) $\beta_{4}$

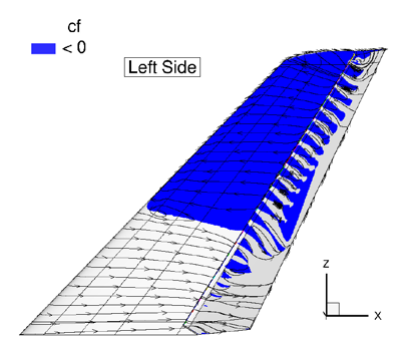

b) $\beta_{5}$
FIG. 17: Skin friction coefficient and stream traces, configuration with blowing, suction side, $\mathrm{v}_{\mathrm{j}}=300 \mathrm{~m} / \mathrm{s}$, discrete slots

\subsection{Variation of the Jet Velocity}

The effects of a variation in the jet velocity will be shown in this section. Due to the large change in the slot area from full span to the discrete slots, this has a large influence on the mass flow of air blown per second over the rudder. In addition to a study on the impact of the jet velocity on the VTP effectiveness, an attempt is made to enable for a comparison of the discrete and continuous slot systems operating at a similar mass flow rate, necessitating different jet velocities in each case.

\subsubsection{Side Force Coefficient}

In FIG. 18 additional curves for the different jet velocities are added to FIG. 14. It can be observed that a smaller jet velocity leads in principal to an offset of the curve to smaller side force coefficients. This is a similar effect on $\mathrm{C}_{\mathrm{S}}$ as if exchanging the continuous slot with discrete slots. For both the mass flow rate is reduced. The change in the side force is due to the change in the rudder suction peak, which gets higher for larger jet velocities, and the effect of the high energy jet on the flow on the rudder, which, for higher jet velocities, leads to a more negative pressure coefficient and thus an increased side force. In addition, the increased rudder suction peak also has an upstream effect in decreasing the pressure coefficient on the fin and increasing the fin leading edge suction peak. This can be seen in FIG. 19.

For the discrete slot results a change in the gradient of the $C_{s}$ vs $\beta$ curve can be observed in FIG. 18 for smaller sideslip angle and the smaller jet velocity. Here the growing regions of separated flow with the decreasing jet velocity and mass flow rate might play a role in leading to a more non-linear flow behavior.

For a jet velocity of $150 \mathrm{~m} / \mathrm{s}$ the continuous jet comes close to the result of the discrete slot configuration with a jet velocity of $300 \mathrm{~m} / \mathrm{s}$. Also for this relatively low jet velocity the continuous slot still shows slightly larger side force coefficients. Nevertheless, these two cases are able to support a comparison for a similar increase in side force coefficient. In the pressure coefficient distribution in FIG. 19 it can be seen that in the chosen mid span section both AFC configurations lead to very similar results on the fin. For the discrete slots result, the rudder shoulder suction peak is decreased since at this position no slot is present. On the rudder, however, this discrete slot configuration features the most negative pressure coefficient on the suction side which exceeds also the curve for the continuous slot. This shows the potential of the discrete slots and their benefit due to the creation of the vortex system on the rudder drawing additional energy from the outer flow towards the rudder surface.

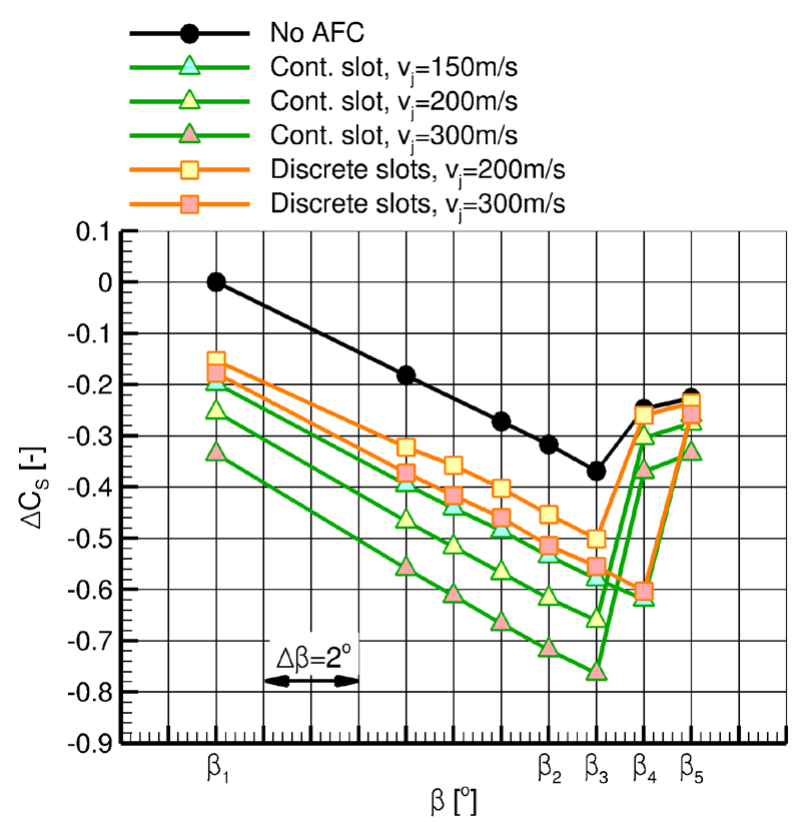

FIG. 18: Side force coefficient versus the sideslip angle, varying jet velocity $v_{j}$

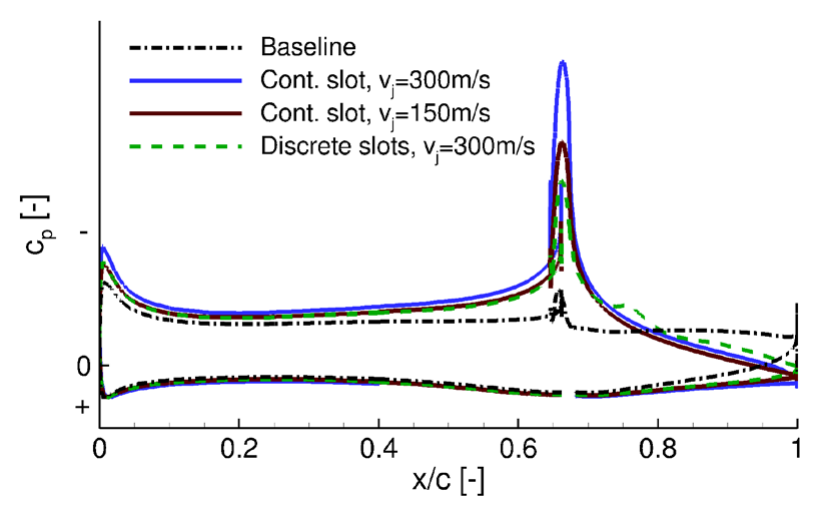

FIG. 19: Comparison of the pressure coefficient distribution for the baseline without blowing and for different jet velocities for the different geometries, section at half span, $\beta_{1}$

\subsubsection{Mass Flow Rate}

For the evaluation of the best approach of using AFC to improve the VTP effectiveness at critical conditions, the maximum achievable increase in the side force coefficient is clearly a very important factor. However, for the aircraft manufacturer it is also important to understand the costs 
involved, i.e. how much mass flow rate is needed, since it has to be provided by some additional system. With this in mind a similar side force coefficient at a lower mass flow rate might be a great benefit. This will be considered in this section.

For the continuous slot and discrete slots configurations calculations are performed for the same AFC jet velocity. The discrete slot configuration, however, has a significantly reduced slot area by $75 \%$. For the same jet velocity this leads to a decrease of the mass flow rate (MFR) of also $75 \%$. This is depicted exemplarily for $\beta_{1}$ in FIG. 20. Due to the linear shift of the curves this looks similar for higher sideslip angles. The subscript of the different mass flow rates describes if it is a constant slot (CS) or discrete slots (DC) and for which jet velocity the depicted data point was obtained. Except from this large benefit concerning the mass flow rate requirement it can be observed that the magnitude of the increase in the side force coefficient is smaller for the discrete slot configuration. An already quite high jet velocity of $300 \mathrm{~m} / \mathrm{s}$ leads to a similar but still somewhat smaller increase in the side force coefficient for the discrete slots than for the continuous slot at a rather small jet velocity of $150 \mathrm{~m} / \mathrm{s}$. If the jet velocity cannot be increased beyond the speed of sound since this may degrade the efficiency of the blowing due to the occurrence of shocks, the jet velocity may have an upper bound. This means that no significant increase in the side force coefficient can be expected by the current discrete slot configuration. Some improvements of the slot distribution might help achieve a further small increase in $\Delta \mathrm{C}_{\mathrm{S}}$ but in general for higher side force coefficients the slot area has to be increased. The continuous slot has the largest slot area and shows the potential of the tangential blowing if the amount of mass flow rate is not limited by some constraints.

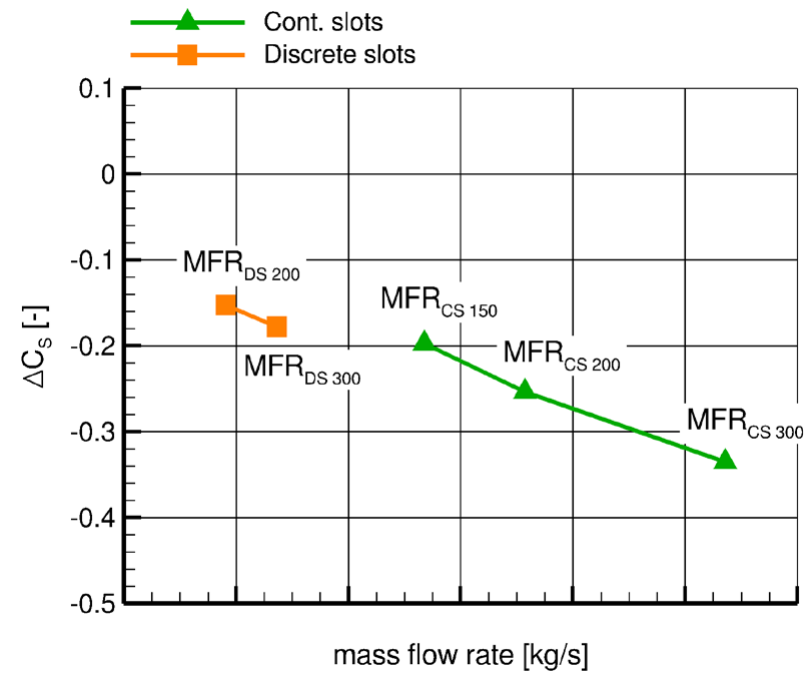

FIG. 20: Side force coefficient increment versus the mass flow rate (MFR) for different jet velocities, $\beta_{1}$

FIG. 21 shows on the vertical axis the increase in the side force coefficient per mass flow rate, i.e. per kilogram of air supply needed in one second. A high magnitude of this value corresponds to a more efficient configuration. Here the discrete slot configuration is clearly more efficient in gaining lift per invested mass flow rate. On the horizontal axis the increase in side force coefficient is depicted. This once again underlines, that the discrete slots are more efficient but also less effective: They cannot be used to achieve the high total $\Delta C_{S}$ which are found for the continuous full span slot. Thus it has to be decided in advance for a particular application which increase in the side force coefficient is required. If $\Delta \mathrm{C}_{\mathrm{S}}<0.18$ is sufficient in this example presented here, the discrete slots would be a good choice. If $\Delta \mathrm{C}_{S}$ of about 0.3 are required however, this can only be realized through the use of the full span slot approach at the cost of a large mass flow rate.

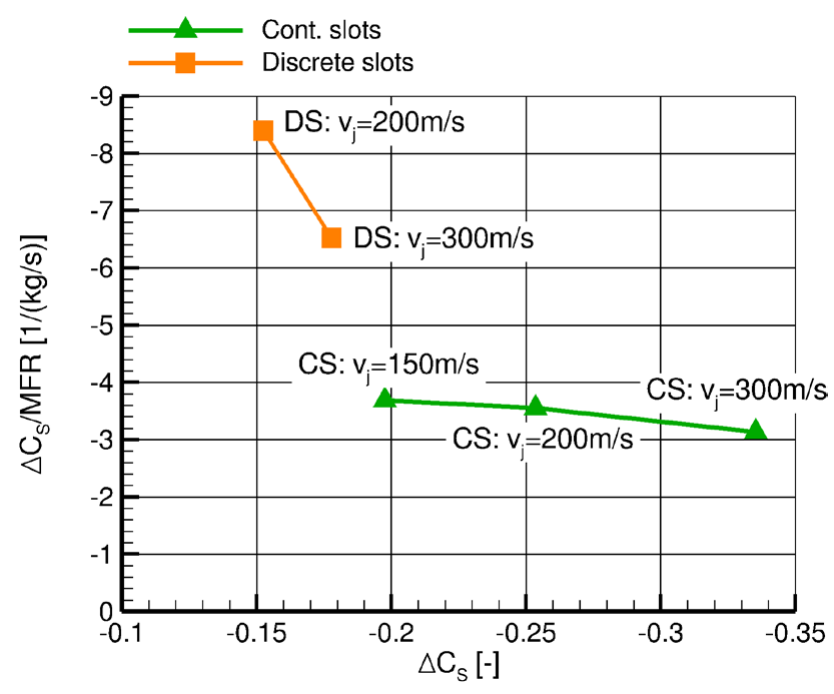

FIG. 21: Ratio of side force coefficient increase per mass flow rate versus the increase in side force coefficient for different jet velocities, $\beta_{1}$

\section{SUMMARY}

A 3D vertical tailplane with large rudder deflection of $30^{\circ}$ is investigated in a numerical study. First the baseline configuration is analyzed, which shows large areas of separated flow on the rudder lowering the achievable VTP side force coefficient. To increase the side force coefficient further, a tangential blowing slot is installed at the end of the fin. First a slot is investigated which covers nearly the whole span. In a next step the continuous full span slot it substituted by several discrete slots.

With tangential blowing it is shown that the flow on the rudder can be held attached. For the continuous slot the flow separation on the rudder can be eliminated completely while for the discrete slots still some small areas of reversed flow exist. Due to this, a significant increase in the side force coefficient can be obtained which is larger for the continuous slot configuration. With increasing sideslip angle the side force coefficient increases approximately linearly. At some sideslip angle a sudden side force breakdown occurs due to a stall of the upper part of the fin. This behavior does not change if blowing is applied or not.

In addition the jet velocity is varied. This leads again to an offset of the side force coefficient versus sideslip angle 
curves. The lower the mass flow rate, the smaller is the achievable increase in the side force coefficient. When looking at the mass flow rate it can be seen that the discrete slots can achieve a given increase in the side force coefficient at a much smaller mass flow rate, allowing for a reduction in the air mass flow of around $50 \%$ for the presented case. However, the obtainable increase in the side force coefficient is limited if the maximum jet velocity is limited. An increase in the mass flow rate can lead to higher gains in the side force coefficient, which can be achieved through the use of a larger slot width in spanwise direction. The continuous slot leads to the largest increase in the side force coefficient but at the expense of a large amount of required mass flow rate.

\section{ACKNOWLEDGEMENTS}

This work is part of the collaborative research project AsSaM (Autoritätssteigerung von Steuerflächen durch aktive Maßnahmen) and funded through the Bundesministerium für Wirtschaft und Energie, which is gratefully acknowledged.

\section{REFERENCES}

[1] K. C. Pfingsten, R. Radespiel: Experimental and numerical investigation of a circulation control airfoil, AIAA Paper 2009-533, 2009

[2] M. Burnazzi, R. Radespiel: Design of a Droopnose Configuration for a Coanda Active Flap Application, AIAA Paper 2013-0487, 2013

[3] N. W. Rathay, M. J. Boucher, M. Amitay, E. Whalen: Performance Enhancement of a Vertical Stabilizer using Synthetic Jet Actuators: No Sideslip, AIAA Paper 2012-0071, 2012.

[4] N. W. Rathay, M. J. Boucher, M. Amitay, E. Whalen: Performance Enhancement of a Vertical Stabilizer using Synthetic Jet Actuators: Non-zero Sideslip, AIAA Paper 2012-2657, 2012.

[5] R. Seele, E. Graft, M. Gharib: Improving Rudder Effectiveness with Sweeping Jet Actuators, AIAA Paper 2012-3244, 2012.

[6] M. Y. Andino, J. C. Lin, A. E. Washburn, E. A. Whalen, E. C. Graff, I. J. Wygnanski: Flow Separation Control on a Full-Scale Vertical Tail Model using Sweeping Jet Actuators, AIAA Paper 20150785, 2015

[7] J. C. Lin, M. Y. Andino, M. G. Alexander, E. A. Whalen, M. A. Spoor, J. T. Tran, I. J. Wygnanski: An Overview of Active Flow Control Enhanced Vertical Tail Technology Development, AIAA Paper 20160056, 2016

[8] R. E. Childs, P. M. Stremel, J. A. Garcia, J. T. Heineck, L. K. Kushner, B. L. Stormsk: Simulation of Sweep-Jet Flow Control, Single Jet and Full Vertical Tail, AIAA Paper 2016-0569, 2016

[9] A. Gebhardt, J. Kirz: "Numerical investigation of slot variations on the efficiency of tangential blowing at a vertical tailplane with infinite span", CEAS Aeronautical Journal, $\mathrm{Vol} 9$, No 1, 2018, DOI 10.1007/s13272-018-0288-1
[10] V. Ciobaca: Validation of Numerical Simulations for Separation Control on High-Lift Configurations, Ph.D. thesis, DLR (German Aerospace Center), 2014.

[11] CentaurSoft: Centaur Hybrid Grid Generation System, [online web site], URL: http://www.centaursoft.com, [cited 20th June 2018].

[12] T. Gerhold: Overview of the Hybrid RANS Code TAU, MEGAFLOW - Numerical Flow Simulation for Aircraft Design, edited by N. Kroll and J. Fassbender, Vol. 89 of Notes on Numerical Fluid Mechanics and Multidisciplinary Design, Springer, 2005, pp. 81-92.

[13] P. Spalart, S. Allmaras: One-Equation Turbulence Model for Aerodynamic Flows, AIAA Paper 92-0439, 1992

[14] M. L. Shur, M. K. Strelets, A. K. Travin, P. R. Spalart: Turbulence Modeling in Rotating and Curved Channels: Assessing the Spalart-Shur Correction, AIAA Journal, Vol. 38, No. 5, 2000, pp. 784-792.

[15] K.-C. Pfingsten, C. Jensch, K. W. Körber, R. Radespiel: Numerical Simulation of the Flow around Circulation Control Airfoils, CEAS 2007-377, 2007 